\title{
Investigating the astrophysical ${ }^{22} \mathrm{Ne}(p, \gamma)^{23} \mathrm{Na}$ and ${ }^{22} \mathbf{M g}(p, \gamma)^{23} \mathbf{A l}$ reactions with a multi-channel scattering formalism
}

\author{
P. R. Fraser ${ }^{1,2, a}$, L. Canton ${ }^{1}$, K. Amos ${ }^{2,3}$, S. Karataglidis ${ }^{3,2}$, J. P. Svenne ${ }^{4}$, and D. van der Kniff ${ }^{2}$ \\ ${ }^{1}$ Istituto Nazionale di Fisica Nucleare, Sezione di Padova, Padova I-35131, Italia \\ ${ }^{2}$ School of Physics, University of Melbourne, Victoria 3010, Australia \\ ${ }^{3}$ Department of Physics, University of Johannesburg, P.O. Box 524 Auckland Park, 2006, South Africa \\ ${ }^{4}$ Department of Physics and Astronomy, University of Manitoba, and Winnipeg Institute for Theoretical \\ Physics, Winnipeg, Manitoba, Canada R3T 2N2
}

\begin{abstract}
The reaction ${ }^{22} \mathrm{Ne}(p, \gamma)^{23} \mathrm{Na}$ is key to the $\mathrm{NeNa}$ cycle of stellar nucleogenesis, and better understanding of the ${ }^{22} \mathrm{Mg}(p, \gamma){ }^{23} \mathrm{Al}$ reaction is needed to understand the ${ }^{22} \mathrm{Na}$ puzzle in $\mathrm{ONe}$ white dwarf novae. We aim to study these reactions using a multi-channel algebraic scattering (MCAS) formalism for low-energy nucleon-nucleus scattering, recently expanded to investigate radiative capture. As a first step towards this goal, we here calculate the energy levels of the mass-23 ( $\mathrm{Ne}, \mathrm{Mg}, \mathrm{Na}, \mathrm{Al}$ ) nuclei. This is not only because the resonant structure of these nuclei are related to the astrophysical $\gamma$-rates of interest, but also because the interaction parameters determined for describing the energy levels are an integral part of the future calculation of the astrophysical reactions when using the MCAS scheme.
\end{abstract}

\section{The ${ }^{22} \mathrm{Mg}(p, \gamma)^{23} \mathrm{Al}$ reaction}

The formation of mass-23 nuclei by radiative capture is of great astrophysical interest. One example is the '22 Na puzzle'. In ONe white dwarf novae, the runaway reaction chain

$$
{ }^{20} \mathrm{Ne}(p, \gamma){ }^{21} \mathrm{Na}(p, \gamma){ }^{22} \mathrm{Mg}\left(\beta^{+}, v\right)^{22} \mathrm{Na}
$$

had been expected to stall due to the long half-life of the decay ${ }^{22} \mathrm{Na} \rightarrow{ }^{22} \mathrm{Ne}^{*}+\beta^{+}+v$. However, the subsequent $\gamma$-ray from the excited ${ }^{22} \mathrm{Ne}$ is not observed, indicating the abundance of ${ }^{22} \mathrm{Na}$ is reduced by some other mechanism. Two proposed competitors to the above chain are

$$
{ }^{20} \mathrm{Ne}(p, \gamma){ }^{21} \mathrm{Na}(p, \gamma){ }^{22} \mathrm{Mg}(p, \gamma)^{23} \mathrm{Al} \quad \text { and } \quad{ }^{20} \mathrm{Ne}(p, \gamma)^{21} \mathrm{Na}(p, \gamma)^{22} \mathrm{Mg}\left(\beta^{+}, v\right)^{22} \mathrm{Na}(p, \gamma)^{23} \mathrm{Mg} .
$$

Direct measurement of the reaction ${ }^{22} \mathrm{Mg}(p, \gamma)^{23} \mathrm{Al}$ is not yet feasible, and though indirect methods have suggested that this reaction does not account for the lack of ${ }^{22} \mathrm{Na}$ in novae ejecta $[1,2]$, it is instructive to investigate the reaction via theoretical means [3-5]. Accordingly, we have begun to determine a ${ }^{22} \mathrm{Ne}(n, n)^{22} \mathrm{Ne}$ interaction with a multi-channel algebraic scattering (MCAS) method [6].

\footnotetext{
a e-mail: prfraser@unimelb.edu.au
} 
Then, using mirror symmetry, we define a potential for ${ }^{22} \mathrm{Mg}(p, p)^{22} \mathrm{Mg}$ scattering, as a first step towards modelling the radiative capture ${ }^{22} \mathrm{Mg}(p, \gamma)^{23} \mathrm{Al}$ using the formalism of [7].

In MCAS, solutions of coupled-channel Lippmann-Schwinger equations are found in momentum space using finite-rank separable representations of an input matrix of nucleon-nucleus interactions. An 'optimal' set of sturmian functions is used as the expansion basis. The MCAS method has the ability to locate all compound system resonance centroids and widths, regardless of how narrow. Also, by use of orthogonalizing pseudo-potentials (OPP) in generating sturmians, it ensures the Pauli principle is not violated [8], even with the collective model formulation of nucleon-nucleus interactions used. Otherwise, some compound nucleus wave functions possess spurious components. The same sturmians determined in the elastic scattering calculations are integral ingredients in the future generation of capture cross sections, as described fully in [7]. MCAS is well suited to modelling the ${ }^{22} \mathrm{Mg}(p, p){ }^{22} \mathrm{Mg}$ reaction due to the low density of low-energy states in ${ }^{23} \mathrm{Al}$ and the low scattering threshold.

The low-lying spectrum of ${ }^{22} \mathrm{Ne}$ consists of a $J^{\pi}=0^{+}$ground state, a $2^{+}$state at $1.274 \mathrm{MeV}$, and a $4^{+}$state at $3.357 \mathrm{MeV}$, which together suggest a collective rotor character. Directly above this comes a $2^{+}$state at $4.456 \mathrm{MeV}$ which decays by E2 transition to the ground state [9]. Thus, a scattering potential based upon the Tamura collective model of rotor character [10] is used to investigate neutron scattering from this target.
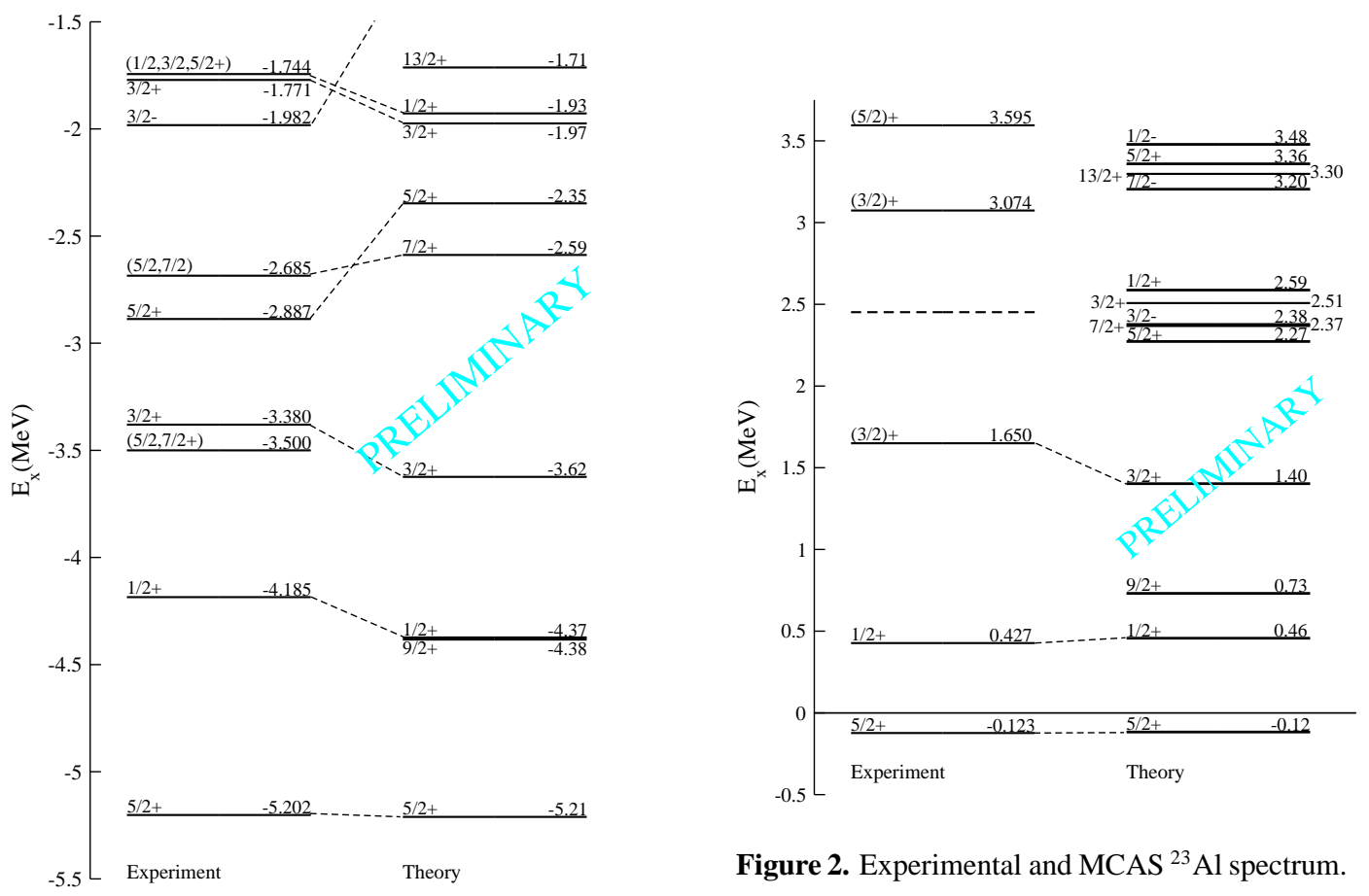

Figure 2. Experimental and MCAS ${ }^{23} \mathrm{Al}$ spectrum.

Figure 1. Experimental and MCAS ${ }^{23} \mathrm{Ne}$ spectrum.

Fig. 1 shows a preliminary theoretical spectrum for ${ }^{23} \mathrm{Ne}$ as compound states of $n+{ }^{22} \mathrm{Ne}$, compared to experimental values. There is still room for improvement, but the low-lying spectrum is reasonably well reproduced. Fig. 2 shows a theoretical spectrum for ${ }^{23} \mathrm{Al}$ as compound states of $p+{ }^{22} \mathrm{Mg}$, 
generated by utilising mirror symmetry and adding a Coulomb potential of the same geometry as the nuclear force. As the nuclear potential is preliminary, the $V_{0}$ depth has been reduced from that of the $n+{ }^{22} \mathrm{Ne}$ potential to allow a better fit to data.

\section{The ${ }^{22} \mathrm{Ne}(p, \gamma)^{23} \mathrm{Na}$ reaction}

Another mass-23 reaction of astrophysical interest is ${ }^{22} \mathrm{Ne}(p, \gamma)^{23} \mathrm{Na}$, currently under experimental investigation by the LUNA collaboration at Gran Sasso. Current stellar models predict that the surface abundance of elements should not change when stars ascend the red giant branch of the HertzsprungRussell diagram of observed stellar luminosity versus temperature. However, anti-correlations have been observed between sodium and oxygen across this branch. An explanation proposed for this is the existence of non-convective mixing that is, possibly, driven by rotation. At high temperatures, this would facilitate leakage from the $\mathrm{CNO}$ cycles into the NeNa cycle, which would produce sodium while depleting oxygen. The reaction that generates sodium in the NeNa cycle is ${ }^{22} \mathrm{Ne}(p, \gamma)^{23} \mathrm{Na}$, a reaction that is not well understood [11]. Better knowledge of it will help to test this idea of mixing.

To evaluate this system using MCAS, the elastic ${ }^{22} \mathrm{Ne}(p, p)^{22} \mathrm{Ne}$ process will first be examined in order to establish an interaction potential, after which radiative capture of the proton will be studied. Given the high proton threshold of ${ }^{23} \mathrm{Na}(8.79 \mathrm{MeV})$, care must be taken in selecting ${ }^{22} \mathrm{Ne}$ states to couple with the proton, such that the deeply-bound states are credibly reproduced as well as compound system states in the Gamow window that contribute to capture (including the first $\frac{1}{2}^{+}$above threshold). Details of these states have been discussed in [12] (see Table 5.9 and discussion).

Fig. 3 shows preliminary low-energy MCAS spectra for the ${ }^{22} \mathrm{Ne}(p, p)^{22} \mathrm{Ne}$ system, and its mirror, ${ }^{22} \mathrm{Mg}(n, n)^{23} \mathrm{Mg}$, using just the four lowest target states. Experimental data are shown for both. Fig. 4 shows the experimental spectrum of ${ }^{23} \mathrm{Na}$ (where red states are assigned positive parity, blue as negative parity, black where no parity has been suggested, and dashed where no $J^{\pi}$ has been suggested), and a partial spectrum of ${ }^{22} \mathrm{Ne}$ (all states up to $6.9 \mathrm{MeV}$, and only states assigned as $2^{+}$above) $[9,12$, 13]. At present, our goal is determining which target states need be included in calculations, as well as their mixings. States under consideration are marked with arrows.

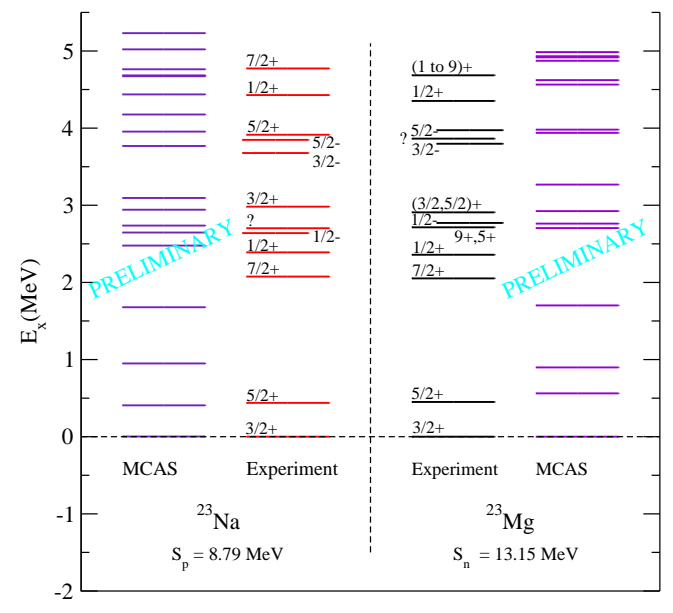

Figure 3. ${ }^{23} \mathrm{Na}$ and ${ }^{23} \mathrm{Mg}$ spectra from experiment and MCAS.

These results, while still preliminary, are promising. The outcomes of this project will guide future MCAS research in other deeply bound systems, including that of the reaction ${ }^{22} \mathrm{Na}(p, \gamma)^{23} \mathrm{Mg}$ mentioned in the first section, where the compound system ground state lies $7.58 \mathrm{MeV}$ below the proton scattering threshold.

\section{References}

[1] T. Al-Abdullah et al., Phys. Rev. C81, 035802 (2010) 
EPJ Web of Conferences


Figure 4. Known spectra of ${ }^{23} \mathrm{Na}$ and ${ }^{22} \mathrm{Ne}$.

[2] A. Banu et al., Phys. Rev. C84, 015803 (2011)

[3] N. Timofeyuk et al., Phys. Rev. Lett. 91, 232501 (2003)

[4] N. Timofeyuk, P. Descouvemont, Phys. Rev. C71, 064305 (2005)

[5] N. Timofeyuk, P. Descouvemont, Phys. Rev. C72, 064324 (2005)

[6] K. Amos et al., Nucl. Phys. A728, 65 (2003)

[7] L. Canton, L. Levchuk, Nucl. Phys. A808, 192 (2008)

[8] L. Canton et al., Phys. Rev. Lett. 94, 122503 (2005)

[9] R.B. Firestone et al., Nucl. Data Sheets 106, 1 (2005)

[10] T. Tamura, Rev. Mod. Phys. 37, 679 (1965)

[11] S.E. Hale et al., Phys. Rev. C65, 015801 (2001)

[12] S.E. Hale, Ph.D. thesis, University of North Carolina at Chapel Hill, Chapel Hill, North Carolina (2012)

[13] R.B. Firestone et al., Nucl. Data Sheets 108, 1 (2007) 